\title{
EVALUACIÓN DE PROPIEDADES FÍSICO-QUÍMICAS Y MECÁNICAS DEL ADOBE ELABORADO CON CAL PARA SU USO EN LA CONSTRUCCIÓN SOSTENIBLE.
}

\author{
ASSESSMENT OF PHYSICAL-CHEMICAL AND MECHANICAL PROPERTIES \\ OF THE ADOBE ELABORATED WITH LIME FOR USE IN SUSTAINABLE \\ CONSTRUCTION.
}

\author{
Ing. Alvaro Doria Alvarez*, MSc. Ing. Javier Orozco Ospino* \\ *Facultad de Ingeniería y Ciencias Básicas, Fundación Universitaria del Área Andina. \\ Trans 22 Bis \# 4 -105, Sector Callejas, Valledupar, Cesar, Colombia. \\ Teléfono: $(+57)(5) 5897879$ \\ E-mail: alvarodorialvarez@gmail.com, jaorozco@areandina.edu.co
}

\begin{abstract}
Resumen: En la actualidad la mejora de los materiales para construcción ha tenido un auge particular hacia la sostenibilidad con el propósito de minimizar el impacto ambiental; esta investigación tiene como eje el diseño de la relación del adobe para mejorar sus propiedades estructurales, fomentando la salud de sus ocupantes y mejorando su calidad de vida, gracias a las características especiales de la arcilla; para ello se realiza un muestreo experimental, con el propósito de llegar a una mejor relación de materiales y a la proporción adecuada, para probar sus otras propiedades habitacionales; aunque en el desarrollo del experimento no se obtienen los resultados de resistencia, es prudente seguir estudiando este tipo de materiales puesto que aún faltan por evaluar muchas de sus características útiles en el campo de la construcción.
\end{abstract}

Palabras clave: adobe, construcción, sostenibilidad, ladrillo, cal.

\begin{abstract}
At present the improvement of materials for construction has had a particular boom towards sustainability with the purpose of minimizing the environmental impact; That is because, this research has as its axis the design of the relationship of the adobe to improve its structural properties, promoting the health of its occupants and improving their quality of life, thanks to the special characteristics of the clay, for it is done a experimental sampling, with the purpose of reaching a better ratio of materials and the appropriate proportion, to test their other housing properties; Although in the development of the experiment the results of the resistance are not obtained, it is prudent to continue studying these types of materials that have not yet been evaluated many of their useful characteristics in the field of construction.
\end{abstract}

Keywords: adobe, construction, sustainability, brick, lime. 


\section{INTRODUCCIÓN}

En la actualidad el desarrollo de la infraestructura es uno de los motores que impulsa el crecimiento de las naciones, por lo que unos buenos materiales y buenas prácticas garantizan la comodidad y seguridad en los inmuebles. En el ámbito de los materiales de construcción quizás el más utilizado por la humanidad son las unidades de mampostería, mejor conocidas como ladrillos; estas unidades actualmente se hacen de diferentes maneras, teniendo en cuenta el alcance de los proyectos y los recursos disponibles. A raíz de esto, no toda la población cuenta con materiales de calidad capaces de garantizar seguridad y comodidad en los inmuebles, siendo los más perjudicados la población rural y los grupos sociales en condición de vulnerabilidad. Por otro lado, la falta de tecnificación de los gremios ladrilleros locales convierte esta actividad en un gran foco de contaminación, puesto que usualmente se talan bosques para emplearlos como combustibles en hornos altamente contaminantes. Algunos de los mampuestos usados se explican a continuación; (L Tangarife et al., 2017).

\section{MAMPOSTERÍA DE ARCILLA COCIDA}

Son piezas formadas generalmente por arcilla y arenas, algunas moldeadas, comprimidas y sometidas a altas temperaturas que luego son distribuidas en el mercado.

Se pueden distinguir dos clases de ladrillos; los que están constituidos de manera cerámica, generalmente resistente, sin fisuras, color uniforme, y por otra parte, los realizados artesanalmente normalmente llamados bocadillo, los cuales son toscos y en ocasiones de coloración dispareja (Franco Moreno G., n.d.); (A Niño, 2018). Tanto las propiedades mecánicas como la constitución de la simetría de cada ladrillo van de acuerdo con su forma de fabricación.

Los ladrillos artesanales requieren de la explotación de depósitos de arcilla que generalmente se consiguen de 4 a 6 metros de profundidad; luego se procede a equilibrar el suelo (además de la arena y la arcilla se usa aserrín y cisco de arroz) con el parámetro de no quedar excesivamente magros o grasos al tiempo en el que se prepara la masa; se usan moldes de madera llamados gradillas que son previamente esparcidos con arena fina con el fin de que la masa no se adhiera al molde y cause malformaciones en los elementos, luego tierra preparada, y se presiona hasta que las gradillas estén totalmente llenas, se quita el excedente con una regla donde son agrupados paralelamente para su secado que dura alrededor de 24 horas, alcanzando algo de tenacidad (Franco Moreno G., n.d.). Luego son cocidos (cochura) en hornos de pampa ${ }^{l} \mathrm{u}$ otros semejantes, con capacidad de 10.000 unidades aproximadamente, donde puede permanecer entre 2 y 6 días dependiendo el volumen del horno y lograr temperaturas de hasta $800{ }^{\circ} \mathrm{C}$, en un proceso donde la llama es alimentada con madera (leña) alrededor de 24 horas y luego se deja enfriar para su distribución (Mario Averardo, 2009); (O Suarez at el, 2018).

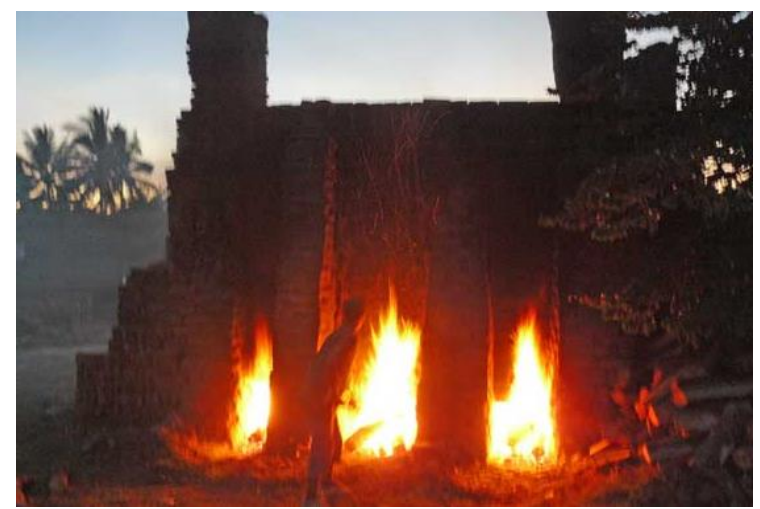

Figura 1. Horno artesanal en forma de pampa

Debido a la contextura rupestre del horno es notorio que la temperatura no es uniforme en cada ladrillo, por lo cual existen márgenes de perdidas grandes, y a la vez una gran variedad de calidades del ladrillo (CORPOCESAR, 2013); (J Plaza, M Núñez, 2017).

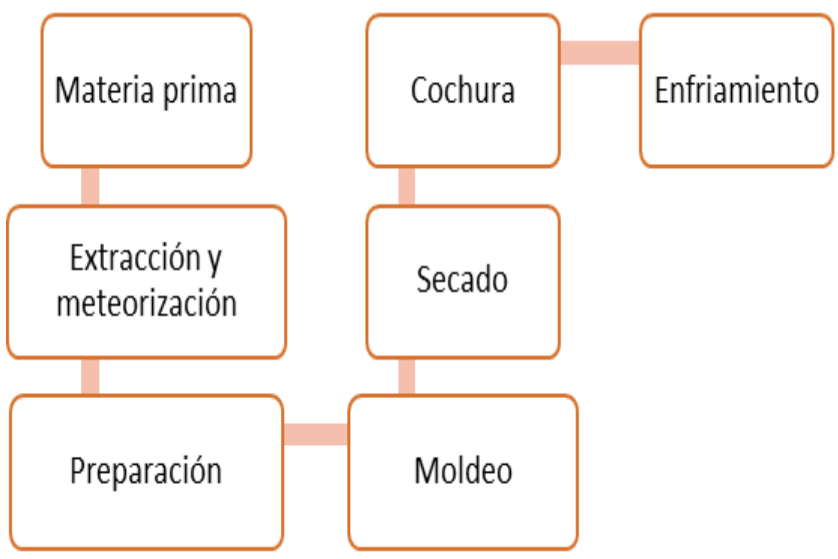

Figura 2. Esquema de la producción de ladrillo de arcilla.

\footnotetext{
${ }^{1}$ Tipo de horno construido con adobes destinados a ser ladrillos.
} 
Tabla 1. Requisitos físicos para ladrillo sólido de arcilla o pizarra

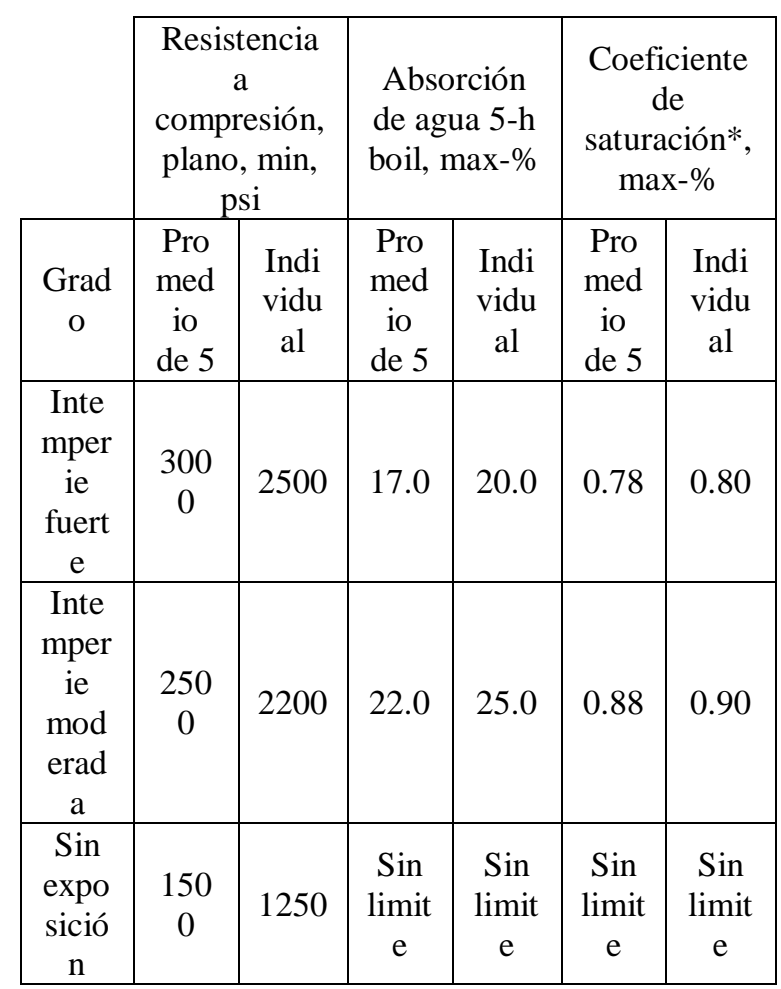

\section{ADOBE}

Es una masa de barro frecuentemente mezclada con paja, moldeada con forma prismática, de tamaño variable y secado al aire para la formación de muros (Johnny Salazar, n.d.).

El adobe como material de construcción para uso habitacional ha sido utilizado durante miles de años por los pueblos indígenas de América, tanto en el suroeste de los Estados Unidos como en Mesoamérica y la región andina en Suramérica. ("Construcción con tierra en el siglo XXI," n.d.).

El adobe generalmente debe tener mayor porcentaje de arena que arcilla. Estos ladrillos, en su comienzo, eran confeccionados a mano, y luego se utilizaban moldes para lograr una mayor producción. La tierra debe permanecer húmeda durante dos días, para fermentarla y lograr que los aglomerantes actúen. Para elaborar el ladrillo, el barro se arroja dentro del molde humedecido, luego se comprime con la mano o los pies, repartiendo uniformemente el material; posteriormente se enrasa la superficie, y se desmolda cuidadosamente para que las aristas permanezcan en buen estado. Si el adobe se raja al secarse es porque presenta mucha arcilla. (Cid Falceto, Ruiz Mazarron, \& Cañas Guerrero, 2011).

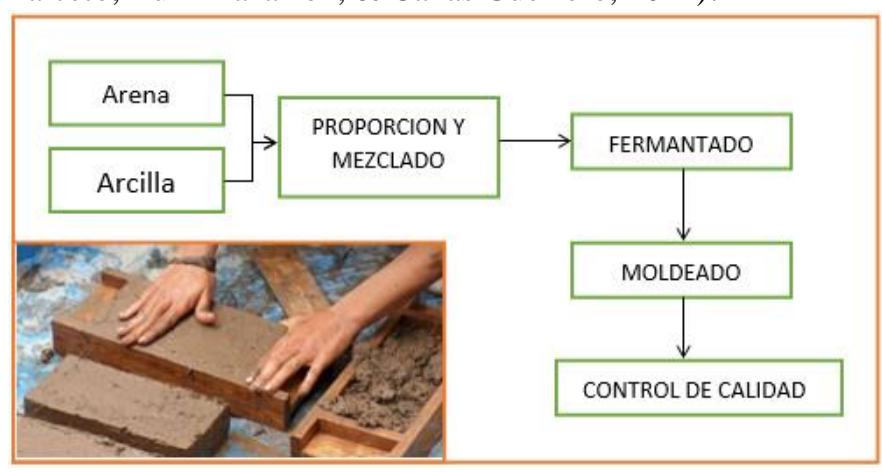

Figura 3. Esquema de fabricación de adobe

Cabe destacar que las unidades de mampostería funcionan soportando y distribuyendo las cargas proporcionadas por el peso de las otras a lo largo del muro, solamente variando su disposición (trabado) para controlar las esfuerzos que reposan sobre cada unidad y sobre su conjunto o muro (Caporale, Parisi, Asprone, Luciano, \& Prota, 2014); (NSR 10, 2010).

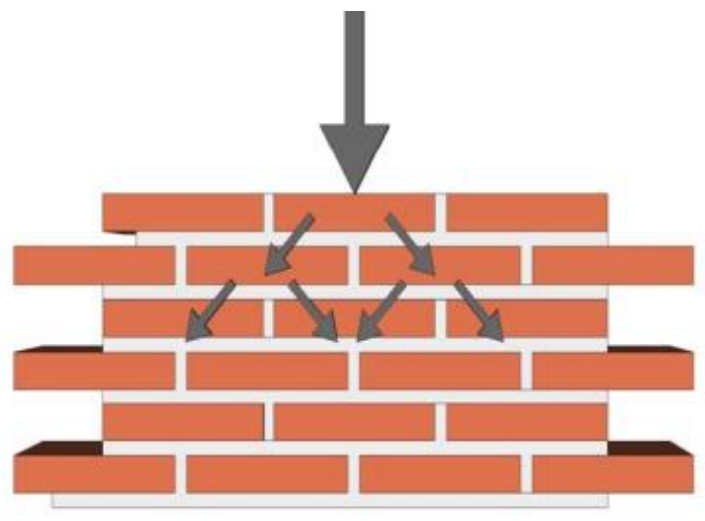

Figura 4. Distribución de cargas mostrando esfuerzos de compresión.

Por todo lo anterior, el eje de esta investigación es mejorar las relaciones de adobe para crear un material de construcción que cumpla con las propiedades mecánicas en la normatividad vigente, puesto que los usuarios del adobe en su mayoría son población vulnerable; esto mejoraría la condición de sus inmuebles, brindando mayor resistencia al agua y otros elementos climáticos y estructurales.

\section{MATERIALES Y MÉTODOS}

En la investigación se empleó la siguiente metodología: 


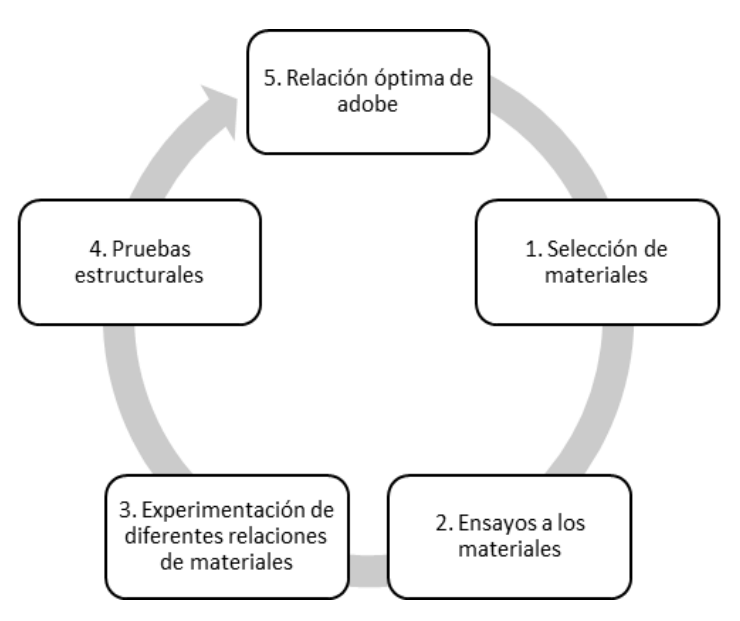

Figura 5. Metodología de investigación

Selección de materiales: la arcilla es procedente de la cantera El Cielo, y la arena de la cantera El Dorado, ambas localizadas en el municipio de Valledupar; la cal hidratada es comercial.

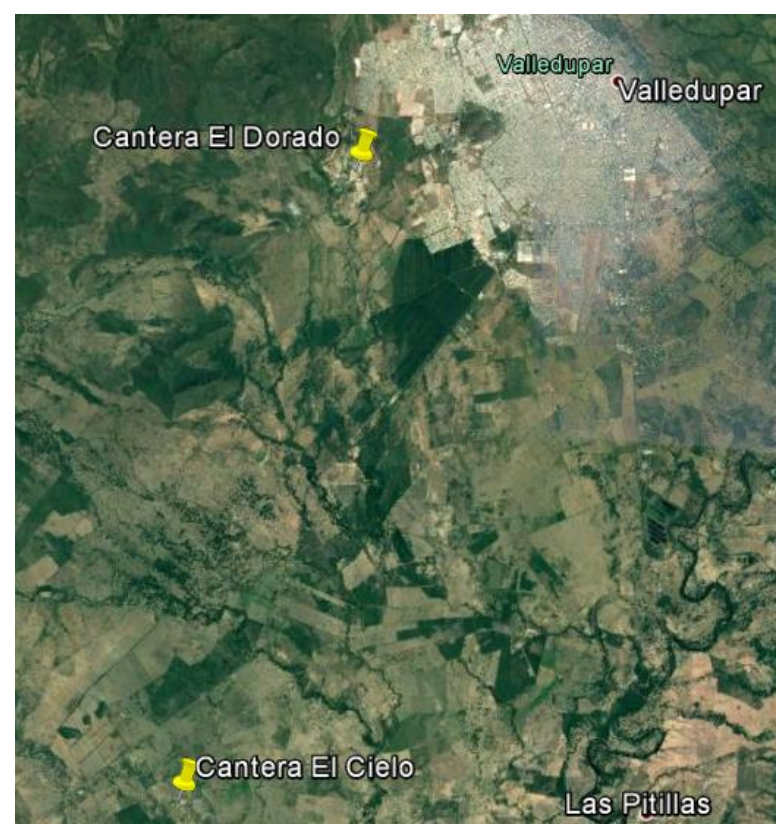

Figura 6. Ubicación geográfica de los puntos de muestreo.

Tabla 2. Coordenadas de los puntos de muestreo.

\begin{tabular}{|l|c|c|}
\hline \multicolumn{1}{|c|}{ Canteras } & Latitud & Longitud \\
\hline El Dorado & $10^{\circ} 27^{\prime} 24.89^{\prime \prime} \mathrm{N}$ & $7^{\circ} 17^{\prime} 46.87^{\prime} \mathrm{O}$ \\
\hline El Cielo & $10^{\circ} 20^{\prime} 00.98^{\prime}{ }^{\prime} \mathrm{N}$ & $7^{\circ} 19^{\prime} 57.30^{\prime \prime} \mathrm{O}$ \\
\hline
\end{tabular}

Ensayos a los materiales: Puesto que la mezcla normal de adobe es una relación de arena y arcilla se debe constatar que estos materiales tengan las propiedades adecuadas, por lo que a cada muestra se le hizo granulometría por tamizado (norma I.N.V. E - 123) e índice de plasticidad a través del método de la cuchara partida (norma I.N.V. E 126).

Experimentación de diferentes relaciones de materiales: se buscó llegar a la mejor relación de cal, arcilla y arena, por medio de una optimización experimental que consiste en cerrar el cerco de incertidumbre probando diferentes relaciones de materiales, analizar los resultados y volver a probar entre los mejores rangos.

Pruebas estructurales: Los adobes se evaluaron mediante su resistencia a la compresión simple. Según la norma I.N.V. E - 410

\section{Relación óptima de adobe:}

Se dosificaron diferentes relaciones volumétricas de los materiales constituyentes del adobe adobe (cal - arcilla - arena) con el fin de determinar la mejor relación a través del ensayo.

\section{RESULTADOS}

Se muestran a continuación los resultados de los ensayos de granulometría e índice de plasticidad de los materiales.

\section{- Arena cantera EI Dorado}

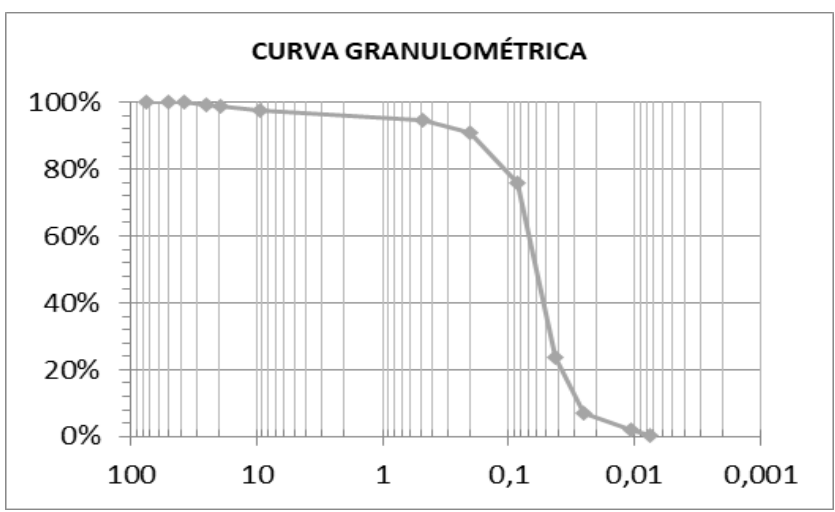

Gráfica 1. Granulometría de la arena de El Dorado.

Tabla3. Índices de la arena de El Dorado.

\begin{tabular}{|l|c|c|c|}
\hline Límite líquido & - & $\begin{array}{c}\text { Índice de } \\
\text { grupo }\end{array}$ & - \\
\hline Límite plástico & - & A.A.S.H.T.O. & A-3 \\
\hline $\begin{array}{l}\text { Índice de } \\
\text { plasticidad }\end{array}$ & NP & U.S.C. & SW \\
\hline
\end{tabular}




\section{- Arcilla cantera El Cielo}

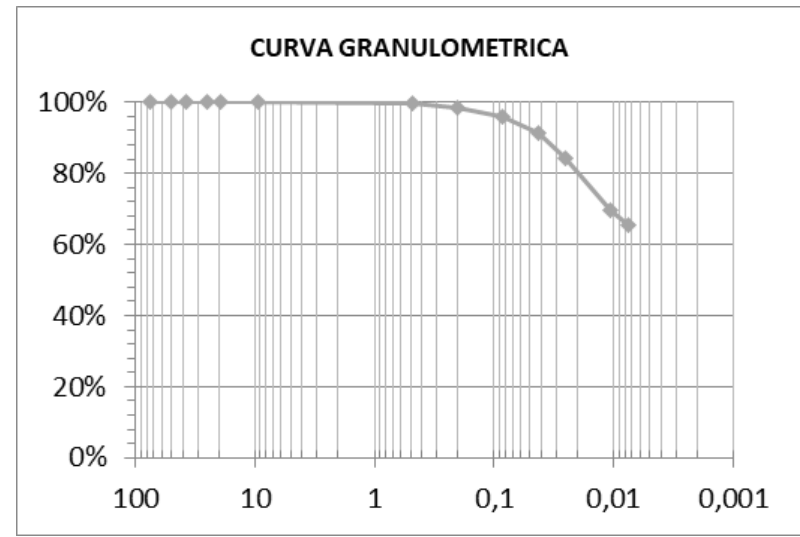

Grafica 2. Granulometría de la arcilla de El Cielo.

Tabla 4. Pruebas índices de la arcilla de El Cielo

\begin{tabular}{|l|c|c|c|}
\hline Límite líquido & 26.3 & $\begin{array}{c}\text { Índice de } \\
\text { grupo }\end{array}$ & 0 \\
\hline $\begin{array}{l}\text { Límite } \\
\text { plástico }\end{array}$ & 15.99 & A.A.S.H.T.O & A-6 \\
\hline $\begin{array}{l}\text { Índice de } \\
\text { plasticidad }\end{array}$ & 10.31 & U.S.C. & CL \\
\hline
\end{tabular}

- A continuación, los resultados de resistencia a la compresión de primera experimentación obtenidos según la metodología planteada. Cabe destacar que, en la relación, los números hacen alusión a los materiales empleados (cal viva: arcilla: arena), siendo relaciones volumétricas.

Tabla 5. Resultados de los especímenes de adobe primera experimentación

\begin{tabular}{|c|c|c|c|c|}
\hline $\begin{array}{c}\text { Relaci } \\
\text { ón }\end{array}$ & $\begin{array}{c}\text { Espécim } \\
\text { en }\end{array}$ & $\begin{array}{c}\text { Días } \\
\text { de } \\
\text { rotur } \\
\mathbf{a}\end{array}$ & $\begin{array}{c}\text { Fuerza } \\
\text { de falla } \\
(\mathbf{K N})\end{array}$ & $\begin{array}{c}\text { Resisten } \\
\text { cia } \\
\text { (Mpa) }\end{array}$ \\
\hline $1: 1: 1$ & 1 & 7 & 11.9 & 0.33 \\
\hline & 2 & 20 & 12.0 & 0.33 \\
\hline & 3 & 28 & 12.1 & 0.33 \\
\hline & & & & \\
\hline $1: 1: 2$ & 1 & 8 & 9.6 & 0.27 \\
\hline & 2 & 14 & 10.5 & 0.29 \\
\hline & 3 & 28 & 11.0 & 0.31 \\
\hline & & & & \\
\hline $1: 1: 3$ & 1 & 7 & 9.0 & 0.25 \\
\hline & 2 & 16 & 10.9 & 0.30 \\
\hline & 3 & 29 & 11.2 & 0.31 \\
\hline
\end{tabular}

\begin{tabular}{|c|c|c|c|c|}
\hline & & & & \\
\hline $1: 2: 1$ & 1 & 9 & 11.6 & 0.32 \\
\hline & 2 & 15 & 12.9 & 0.36 \\
\hline & 3 & 28 & 13.0 & 0.36 \\
\hline & & & & \\
\hline $1: 2: 2$ & 1 & 6 & 10.5 & 0.29 \\
\hline & 2 & 20 & 11.1 & 0.31 \\
\hline & 3 & 28 & 11.6 & 0.32 \\
\hline & & & & \\
\hline $1: 2: 3$ & 1 & 7 & 8.4 & 0.23 \\
\hline & 2 & 21 & 10.8 & 0.30 \\
\hline & 3 & 28 & 10.9 & 0.30 \\
\hline
\end{tabular}

Al analizar los resultados primarios se decide finalizar el experimento puesto que se alejan mucho del valor de $10 \mathrm{MPa}$ exigido por la norma NSR 10, por lo que se puede concluir que los adobes no cumplen con el mínimo de resistencia exigidos por ley.

\section{DISCUSIÓN}

De los resultados podemos ver que las relaciones con más contenido de arcilla tuvieron mejor comportamiento respecto a las demás muestras, por lo que se presume que los adobes estabilizados con cal necesitan algún catalizante físico o químico que mejore la relación. Respecto a sus propiedades diferentes a la resistencia, no se realizaron puesto que el ladrillo no tiene utilidad estructural.

Al guiarse de los ensayos emulando los estudios para concreto no se contempló en esta investigación un desarrollo de resistencia después de los 28 días, por lo que pudo existir sesgo, en el periodo de ensayo de las muestras.

En la relación propuesta se usó la cal hidratada, la cual no sufre transformaciones químicas que afecten a los materiales en cuestión. Se recomienda usar para próximos experimentos cal viva, precisamente porque su inestabilidad con el agua limitaría la absorción de esta en la arcilla, y al carbonatarse pueden producir un elemento más resistente.

\section{BIBLIOGRAFIA}

AB Niño (2018) Micro turbina Peltón, una solución real de energía para zonas no interconectadas (ZNI). Revista Colombiana 
de Tecnologías de Avanzada, ISSN: 16927257.

Brotóns, P. U. (2006). Construcción de estructuras de hormigón armado: adaptado a las instrucciones EHE, EFHE, NCSE-02 y CTE. Editorial Club Universitario.

Caporale, A., Parisi, F., Asprone, D., Luciano, R., \& Prota, A. (2014). Micromechanical analysis of adobe masonry as two-component composite: Influence of bond and loading schemes. Composite Structures, 112, 254263. doi:10.1016/j.compstruct.2014.02.020

Cid Falceto, J., Ruiz Mazarron, F., \& Cañas Guerrero, I. (2011). Las normativas de construcción con tierra en el mundo. Informes de la construccion revista de informacion tecnica, 63(523), 159-169.

Construcción con tierra en el siglo XXI. (n.d.). julio-septiembre 2011, 63, 523, 5-20, doi:10.3989/ic. 10.046

CORPOCESAR. (2013). Diagnostico sectorial de la industria ladrillera (informe tecnico) (p. 32). Valledupar.

Corral, J. T. (2008). El Suelo-Cemento Como Material De Construcción. Ciencia y Sociedad, XXXIII(4), 520-571.

Franco Moreno G. (n.d.). Tecnicas De La Construccion Con Ladrillo. peru: CEAC S.A.

Frederick S. Merritt, Loftin, M. K., \& Jonathan T. Ricketts. (2001). Manual del Ingeniero Civil (Vols. 1-3, Vol. 1). MEXICO: McGRAWHILL/INTERAMERICANA EDITORES, S.A.

Gobernación del Cesar. (2012). Estudio de factibilidad al proceso de reconversión de la producción en la industria del ladrillo en los municipios de Valledupar y San Diego del departamento del Cesar. municipios de Valledupar y San Diego del departamento del Cesar: Gobernacion del cesar.

JEG Plaza, MAR Nuñez, (2017) Formación en competencias específicas para la industria del software colombiano. Experiencias del uso del aprendizaje basado en proyectos. Revista Colombiana de Tecnologías de Avanzada, ISSN: 1692-7257

Johnny Salazar. (n.d.). manual de construcción natural construyendo con cop. Retrieved from http://www.ecohabitar.org/wpcontent/uploads/2013/10/Manual-deconstruccio\% CC\%81n-natural-Construyendocon-Cob..pdf

L Tangarife, M Sánchez, M Rojas (2017). Modelo de interventoría de tecnologías de información en el área de conocimiento de la gestión del alcance de PMBOK® y alineado con ISO 21500 y COBIT®. Revista

Colombiana de Tecnologías de Avanzada, ISSN: 1692-7257.

La Guia Completa sobre Albanileria y Mamposteria: Incluye trabajos decorativos en Concreto. (2010). Creative Publishing Int'l.

Mario Averardo. (2009). EL LADRILLO (Vols. 16, Vol. 1). CHACO, ARGENTINA.

Milton, H. J., Standards, U. S. N. B. of, \& Standards, U. S. D. of E., Building Technology, and. (1980). International and national standards on dimensional coordination, modular coordination, tolerances and joints in building. U.S. Dept. of Commerce, National Bureau of Standards.

Nagaraj, H. B., Sravan, M. V., Arun, T. G., \& Jagadish, K. S. (n.d.). Role of lime with cement in long-term strength of Compressed Stabilized Earth Blocks. International Journal of Sustainable Built Environment. doi:10.1016/j.ijsbe.2014.03.001

O Suarez, C Vega, E Sánchez, A Pardo. (2018) Degradación anormal de p53 e inducción de apoptosis en la red P53-mdm2 usando la estrategia de control tipo pin. Revista Colombiana de Tecnologías de Avanzada, ISSN: 1692-7257

REGLAMENTO COLOMBIANO DE CONSTRUCCIÓN SISMO RESISTENTE, Pub. L. No. Ley 400 de 1997 (2010). 